\title{
A NEW INSTRUMENT TO MEASURE THE SURFACE RESISTANCE OF SUPERCONDUCTING SAMPLES AT $400 \mathrm{MHz}$
}

\author{
E. Mahner, S. Calatroni, E. Chiaveri, E. Haebel, J.M. Tessier
}

\begin{abstract}
A $400-\mathrm{MHz}$ niobium quadrupole resonator has been manufactured to study the rf properties of superconducting bulk and thin film samples at low temperatures. We describe the apparatus, i.e. the construction of the resonator, field calculations with MAFIA, and the experimental procedure. In first validation tests the surface resistance $R_{s}$ of a reactor-grade bulk niobium sample as a function of temperature and applied rf field has been investigated by using a calorimetric "rf-dc-compensation" method. A critical temperature $T_{c}=9.15 \pm 0.02 \mathrm{~K}$, a thermal conductivity $\lambda(4.2 \mathrm{~K})=6.9 \pm 0.7 \mathrm{~W} / \mathrm{mK}$, a residual resistance $R_{\text {res }}=19.0 \pm 0.3 \mathrm{n} \Omega$ and a superconducting energy gap of $\Delta / k_{B} T_{c}=1.82 \pm 0.01$ have been measured. At $4.2 \mathrm{~K}$ we achieved a calorimetric detection limit for $R_{s}$ of $0.16 \mathrm{n} \Omega$ at a peak field of $25 \mathrm{mT}$.
\end{abstract}

AB, AT, EST Divisions

To be published in Review of Scientific Instruments

Administrative Secretariat AT Division CERN

CH - 1211 Geneva 23
Geneva, Switzerland

4 April 2003 


\section{Introduction}

Superconducting cavities used in particle accelerators are usually operated in the $\mathrm{TM}_{010}$ mode, and their measured unloaded quality factor allows the calculation of the surface resistance. However, in this mode, the surface resistance obtained is actually the average of the surface resistances that different surface regions of the cavity may have. Although inessential for operation in a particle accelerator, this averaging process results in added complications when optimising the manufacturing process of cavities that additional instrumentation cannot completely solve. As an example we mention the difficulty to optimise the chemical preparation for all surface regions of a cavity, independent of its type, bulk niobium or niobium-coated copper. An issue particularly important for niobium-coated cavities is the dependence of the film performance on the impact angle of the niobium atoms on the copper surface. This angle is known to influence the film structure ${ }^{1}$, but its effect on the surface resistance cannot be identified with cavities.

Until now rf tests of samples have been restricted to $\mathrm{TE}_{011}$ resonators where the end plate can be demounted and undergo different treatments ${ }^{2}$. However, these resonators have the disadvantage of a comparatively large size. At $400 \mathrm{MHz}$, the frequency chosen for the LHC collider at CERN, the sample disk would have a diameter of about $1.1 \mathrm{~m}$ ! A first attempt to solve the size problem was realised with the triaxial cavity at CEBAF and CERN ${ }^{3,4}$. This device introduced a novel technique for the determination of the surface resistance: It measures the heat generated in the test sample by rf dissipation in substituting it by heat from dc dissipation. However, since the sample disk is placed at a maximum of the electric field - with the concomitant problem of enhanced field emission - the field ratio $E(\mathrm{MV} / \mathrm{m}) / B(\mathrm{mT})$ is even more unfavourable than in an accelerating cavity. A second problem is that the rf current passing the sample edge creating uncontrollable spurious dissipative effects.

A new device called quadrupole resonator is described in this paper. Its design avoids these two major problems of the triaxial cavity but adopts the thermal substitution technique to measure the surface resistance of superconducting samples.

\section{Some basic cavity notions}

An important quantity of a superconducting cavity is the "unloaded" quality factor $Q_{0}$ which is defined as the ratio between the stored energy $U$ and the dissipated power $P_{\text {diss }}$ lost per radian and second $(\omega=2 \pi f)$ in the cavity walls.

$$
Q_{0}=\frac{\omega U}{P_{\text {diss }}}=\frac{G_{c}}{R_{S}}
$$

The total stored energy in the cavity is given by

$$
U=\frac{1}{2} \mu_{0} \int_{\text {Vol. }}|H|^{2} \times d v=\frac{1}{2} \varepsilon_{0} \int_{\text {Vol. }}|E|^{2} \times d v
$$

where one integrates over the volume of the cavity. $H$ and $E$ are the amplitudes of the magnetic and electric field, respectively. The dissipated power is related to the surface resistance $R_{\mathrm{s}}$, and assuming that the latter does not vary over the inner cavity surface, one can show that 


$$
\begin{aligned}
& P_{\text {diss }}=\frac{1}{2} \int_{\text {Surf. }} R_{S} \times|H|^{2} \times d s \equiv \frac{1}{2} R_{S} \int_{\text {Surf. }}|H|^{2} \times d s \\
& P_{\text {diss }}=\frac{1}{2} R_{S} \times S \times H^{2},
\end{aligned}
$$

where the integration is carried out over the cavity surface. $P_{\text {diss }}$ is proportional to $R_{\mathrm{s}}$ and the surface area $S$. The geometry factor $G_{\mathrm{c}}$ depends only on the electromagnetic field configuration and therefore on the shape but not on the size of the cavity:

$$
G_{c}=\frac{\omega \mu_{0} \int_{\text {Vol. }}|H|^{2} \times d v}{\int_{\text {Surf. }}|H|^{2} \times d s} .
$$

In contrast to the unloaded quality factor $Q_{0}$, a superconducting cavity with couplers is characterised by a "loaded" quality factor

$$
Q_{L}=\frac{\omega U}{P_{\text {total }}}=\omega \tau_{L} .
$$

If one turns off the rf, the energy in the superconducting cavity decays exponentially with the time constant $\tau_{\mathrm{L}}$. The total power dissipation $P_{\text {total }}$ in the cavity is then given by

$$
P_{\text {total }}=\sum_{i=0}^{4} P_{i}=P_{\text {diss }}+P_{1}+P_{2}+P_{\text {beam }}+P_{\text {mult }, \text { efe }}
$$

where $P_{1}$ and $P_{2}$ are losses in the couplers, $P_{\text {beam }}$ are losses induced in the presence of a beam. The term $P_{\text {mult,efe }}$ describes the losses due to multipacting and enhanced field emission in the cavity. In fact, in today's superconducting cavities used in R\&D studies, as well as in accelerators, the enhanced field emission is one of the major limitations to reach higher gradients. From the equations (5) and (6) we see that

$$
\frac{1}{Q_{L}}=\frac{1}{\omega U} \sum_{i=0}^{4} P_{i}=\sum_{i=0}^{4} \frac{1}{Q_{i}}
$$

where $Q_{1}$ and $Q_{2}$, attributed to the input and output coupler, are called external quality factors $\left(Q_{\text {ext }}\right)$. Without any beam $\left(Q_{3} \rightarrow \infty\right)$ and no anomalous losses $\left(Q_{4} \rightarrow \infty\right)$ one obtains, for a strongly overcoupled resonator with equal input and output coupling $\left(Q_{1}=Q_{2}<<Q_{0}\right)$, the following relation

$$
Q_{1}=Q_{2} \approx 2 Q_{\mathrm{L}}=2 \omega \tau_{\mathrm{L}}
$$

With equation (5) we find the following simple linear relation between the stored energy $U$, the time constant $\tau_{\mathrm{L}}$, and the output power $P_{2}$ which is often called (in cavity work) transmitted power $P_{\mathrm{t}}$ :

$$
U=2 \tau_{\mathrm{L}} P_{\mathrm{t}} .
$$




\section{Design of the apparatus}

\subsection{Quadrupole resonator}

The niobium quadrupole cavity consists of three separate parts, as shown in Figure 1. A $2 \mathrm{~mm}$ thick niobium cylinder $(\varnothing 210 \mathrm{~mm}, \ell=361 \mathrm{~mm})$ is composed of two separate niobium cans (parts $1 \& 2$ ) which are electron beam welded and vacuum brazed to stainless steel flanges. This solution allows efficient cleaning, optical inspection and easy handling of the resonator. Inside this screening container, a four-wire transmission line built from niobium rods $(\varnothing 16 \mathrm{~mm})$ is welded to the upper cover plate of the resonator. Special mechanical supports are provided to assure colinearity of the resonator rods with the vertical axis of the niobium cylinder. At the bottom end of the rods the niobium tubes are bent to form half rings of $25 \mathrm{~mm}$ radius. The resulting loop "illuminates" the front disk $(\varnothing 75 \mathrm{~mm})$ of the investigated sample, which is positioned $1 \mathrm{~mm}$ below the niobium half rings. This distance is controlled by measuring the quadrupole resonator frequencies of different modes at room temperature. The test cylinder is fixed to a 6 inch Conflat flange (part 3) and mounted into a tubular port of $2 \mathrm{~mm}$ bigger radius and equal length to ensure that the sample surface flushes with the lower cover plate of the niobium cylinder to which the port is welded. Basically, resonance of the niobium resonator is established because short circuits have been placed in two planes at a distance of about $\lambda / 2$. One short is the upper coverplate of the niobium enclosure, the other short is located at the bottom part of the niobium rods where they are welded to form the two half rings. It is important to notice that the two flanges of the niobium can are positioned at about $\lambda / 4$ where the screening current of the quadrupole resonator vanishes. Regarding the niobium rods as the conductors of a screened four-wire transmission line excited in its quadrupole mode, we have named the apparatus quadrupole resonator. The choice of this mode is most important for the functioning of the instrument. In fact, within the cylindrical gap between sample cylinder and sample port this mode is in cut off and the rf field amplitude decays with approximately 4 to $5 \mathrm{~dB} / \mathrm{cm}$.

\subsection{Sample thermometry}

The sample is a $4 \mathrm{~mm}$ thick reactor-grade niobium disk $(\varnothing 75 \mathrm{~mm})$ electron beam welded to a niobium cylinder ( $\varnothing 75 \mathrm{~mm}, \ell=110 \mathrm{~mm}, 2 \mathrm{~mm}$ thick) which has been welded to a 6-inch Conflat flange. The front disk of the sample carries, on its inner side (under vacuum), three small stubs where temperature sensors and a heater are mounted (see Fig. 1). To vary and measure the temperature of the sample front disk, a metal film resistor $(1 / 8 \mathrm{~W}, 1 \mathrm{k} \Omega)$ is glued into a special copper support which is screwed with Apiezon $\mathrm{N}$ grease to the central stub $\varnothing 10 \mathrm{~mm}, \ell=8$ $\mathrm{mm}$ ). The four Manganin wires of the resistor are thermally anchored over a length of $20 \mathrm{~mm}$ to the copper support. The power delivered to this heater assembly is controlled by a dc current source (HP6186C) and a voltmeter.

For absolute and relative temperature measurements of the sample disk, two calibrated (1.4-100 K) silicon diodes (DT-470-SD, LakeShore) are screwed with Apiezon N grease to the two other stubs $(\varnothing 8 \mathrm{~mm}, \ell=8 \mathrm{~mm})$ at the backside of the sample. In order to measure the temperature of the sample cylinder, up to eight additional thermometers can be fixed onto a mechanical springloaded system connected to a Teflon holder which is screwed onto a $6-4 \frac{1}{2}$ inch Conflat adapter flange. A 55-pin instrumentation feedthrough (Ceramaseal) has been welded into a modified 4$1 / 2$ inch Conflat flange where a pumping port is horizontally attached. 


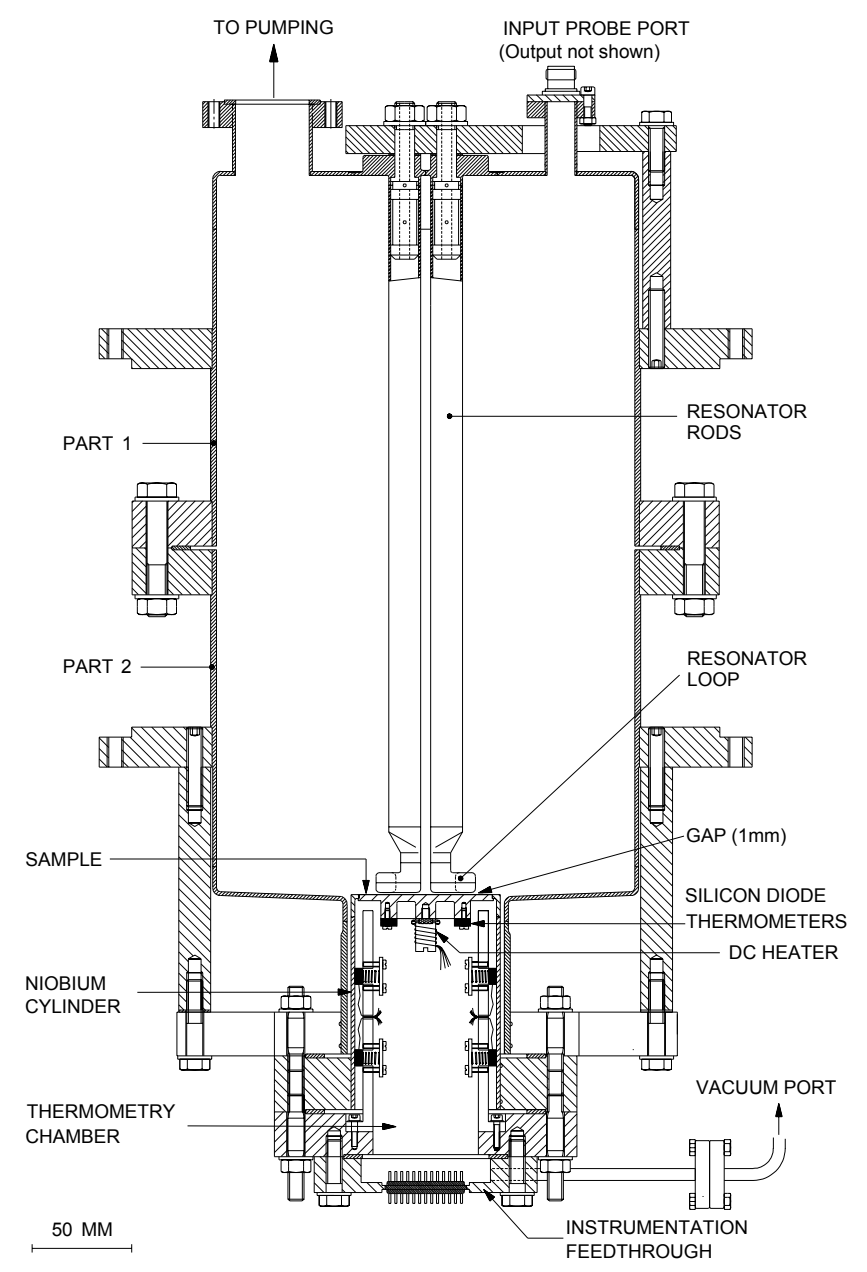

Figure 1: Layout of the niobium quadrupole resonator with mounted sample cylinder and thermometry chamber housing a dc heater and temperature sensors.

A data acquisition unit (HP3497A) provides a current of $10 \mu \mathrm{A}$ used for the silicon diodes and measures all sensor voltages with a resolution of $100 \mu \mathrm{V}$ in the $2 \mathrm{~V}$ range of its incorporated digital voltmeter. At $1.8 \mathrm{~K}$, which is currently the lowest sample temperature we reached, the resistance of the silicon diodes is approximately $1.7 \mathrm{M} \Omega$ and their sensitivity $\Delta R / \Delta T$ is about $1.87 \Omega / \mathrm{mK}$.

\subsection{Calculations of the electromagnetic field distribution in the quadrupole resonator}

A three-dimensional cavity code (MAFIA) has been used to study the field distribution inside the quadrupole resonator. A particular problem was to find a mesh configuration fine enough to resolve accurately the fields between the loop and the sample surface without exceeding the maximally allowed number of mesh points. Finally, the resonator volume was split at the main 


\begin{tabular}{ll}
\hline \hline \multicolumn{1}{c}{ Parameter } & Quadrupole Mode \\
\hline$f$ & $402.9544 \mathrm{MHz}$ \\
$G_{\text {sample }}$ & $215 \Omega$ \\
$U$ & $3.55 \times 10^{-11} \mathrm{~J}$ \\
$E_{\max }($ sample $) / B_{\max }($ sample $)$ & $0.053 \mathrm{MV} / \mathrm{m} / \mathrm{mT}$ \\
$E_{\max }(\lambda / 4) / B_{\max }($ sample $)$ & $0.26 \mathrm{MV} / \mathrm{m} / \mathrm{mT}$ \\
$B_{\max }($ cover $) / B_{\max }($ sample $)$ & 0.86 \\
\hline
\end{tabular}

Table 1: MAFIA calculated rf parameters for the niobium quadrupole resonator.

flange (at $\lambda / 4$ from the upper cover plate) and a magnetic boundary was defined there. Field matching at this boundary served to obtain the complete solution. By using different boundary conditions on the two perpendicular symmetry planes, passing through the axis of the cylinder, one finds in addition to the quadrupole mode a monopole and a dipole mode. The nearest mode is the dipole mode at $10 \mathrm{MHz}$ higher frequency. The monopole mode is more than $250 \mathrm{MHz}$ below. To avoid any difficulty due to a spurious excitation of the dipole mode, the coupling loops have a position and orientation which suppresses coupling to that mode. The sample geometry factor $G_{\text {sample }}$ is calculated by MAFIA using equation (4), but with the surface integral extended only over the sample surface. The most important MAFIA results are summarised in Table 1.

It should be noted that the layout of the quadrupole resonator differs radically from the approach of the triaxial cavity design ${ }^{3,4}$ where the maximum of the E-field coincides with the centre of the sample. In addition, the average magnetic field $\left\langle H_{\mathrm{rf}}(r)\right\rangle$ on the sample surface has been calculated for a normalised stored energy of $U=1 \mathrm{~mJ}$ as well as the dissipated power $P_{\text {diss }}(r)$. Both results are shown in Figure 2.

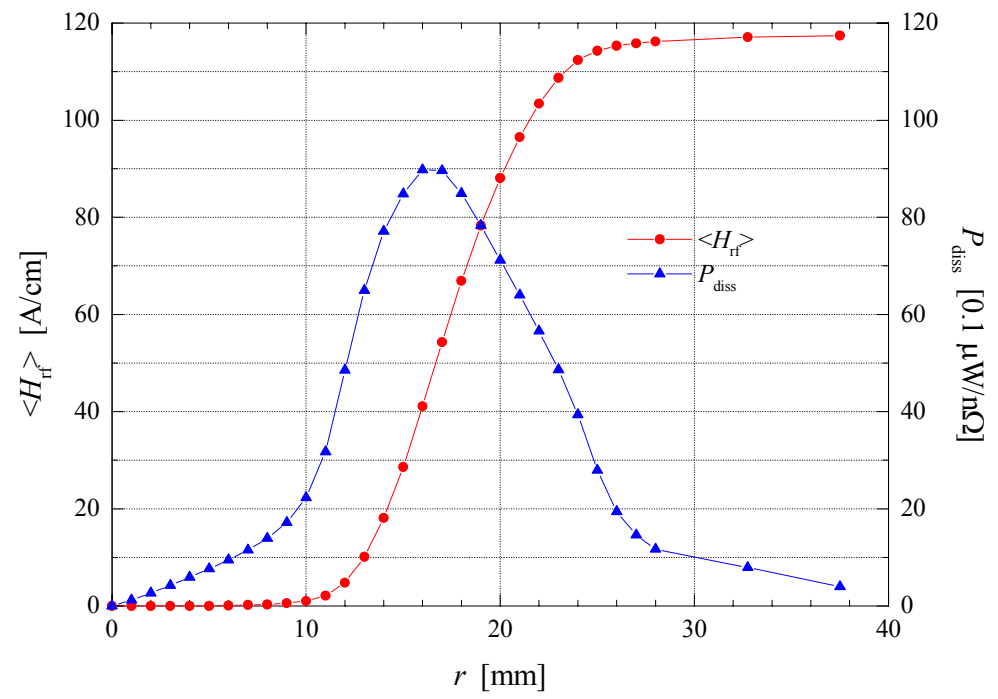

Figure 2: Average magnetic field $\left\langle H_{\mathrm{rf}}>\right.$ and dissipated power $P_{\text {diss }}$ on the sample surface vs. radius $r$ calculated with MAFIA for a stored energy of $U=1 \mathrm{~mJ}$. 


\subsection{Determination of the surface resistance}

In order to calculate the surface resistance of the test sample that is mounted at the bottom of the quadrupole resonator, one can apply the same formalism as described in chapter 2 . Since the dissipation is measured by calorimetry, no determination of $Q_{0}$ is needed. Therefore, the coupling to the resonator is realised by two strongly over-coupled $(\beta \approx 100)$ loops, which are mounted on the upper cover plate. This allows the approximation $P_{\text {total }}=2 P_{\mathrm{t}}$. Taking

$Q_{\text {sample }}=\frac{\omega U}{P_{\text {diss }}}=\frac{G_{\text {sample }}}{R_{S \text {,sample }}}$ one finds with the equations (5) and (7) the final result for the sample surface resistance inside the quadrupole resonator:

$$
R_{S, \text { sample }}=\frac{G_{\text {sample }}}{\omega \tau} \times \frac{P_{\text {diss }}}{P_{\text {total }}} \cong \frac{G_{\text {sample }}}{2 \omega \tau} \times \frac{P_{\text {diss }}}{P_{t}} .
$$

Using the equations (3) and (10), the average magnetic field $\left\langle H_{\mathrm{rf}}\right\rangle$ on the sample surface can be written as

$$
\left\langle H_{r f}^{2}\right\rangle=2 \frac{\omega \tau}{G_{\text {sample }}} \times \frac{P_{\text {total }}}{S_{\text {ill }}} \cong 4 \frac{\omega \tau}{G_{\text {sample }}} \times \frac{P_{t}}{S_{\text {ill }}} .
$$

There was a small arbitrariness in the choice of the "illuminated" sample surface $S_{\text {ill }}$. Finally, we took the stripline's projected surface on the sample surface, which results in $S_{\mathrm{ill}} \cong 12 \mathrm{~cm}^{2}$.

According to the microscopic theory of Bardeen-Cooper-Schrieffer ${ }^{5}$ (BCS) the temperature and frequency dependence of the surface resistance can be expressed with negligible errors for $T<$ $T_{\mathrm{c}} / 2 \mathrm{as}^{6}$

$$
R_{B C S} \propto \frac{\omega^{2}}{T} \times \exp \left(-\frac{\Delta}{k_{B} T_{c}}\right) .
$$

The BCS theory predicts a proportionality between the energy gap $\Delta$ and the critical temperature $T_{\mathrm{c}}$ of the superconductor, namely $\Delta / k_{\mathrm{B}} T_{\mathrm{c}}=1.75$ ( $k_{\mathrm{B}}$ : Boltzmann constant). In many experiments the measured surface resistance at low temperature was higher than the BCS prediction and limited by a temperature independent residual resistance $R_{\text {res }}$. Therefore equation (12) was modified to

$$
R_{S}(T)=R_{B C S}(T)+R_{r e s} .
$$

A summary of theoretical and experimental studies regarding the origin of $R_{\text {res }}$ can be found elsewhere?. 


\section{Experimental results}

\subsection{Sample preparation}

A bulk $\mathrm{Nb}$ sample made from sheets with a nominal residual resistivity ratio (RRR) of about 30 was prepared for commissioning and testing of the quadrupole resonator. The chosen niobium purity should represent a good compromise between reaching a high resolution in the measurement of the surface resistance, and having rf fields intense enough without overheating of the sample.

A surface damage layer of about $100 \mu \mathrm{m}$ was removed by buffered chemical polishing (BCP) in a 1:1:2 solution of $\mathrm{HF}(49 \%), \mathrm{HNO}_{3}(69 \%)$, and $\mathrm{H}_{3} \mathrm{PO}_{4}(85 \%)$. For the preparation of the sample and the resonator, special precautions were taken to avoid, as much as possible, particle contamination on the niobium surfaces which could stimulate enhanced field emission during operation. Therefore, at the end of the polishing, the BCP was instantly diluted with ultra-pure water to avoid any exposure of the niobium to air before the standard high-pressure ultra-pure water rinsing and the final nitrogen gas drying were performed. A detailed description of this advanced preparation technique - which is known to suppress field emission (E $>100 \mathrm{MV} / \mathrm{m})$ of niobium and copper samples - can be found elsewhere ${ }^{8}$.

\subsection{Results and discussion}

After preparation and sample mounting, the quadrupole cavity and the thermometry chamber were evacuated and installed in one of the liquid helium cryostat used for testing LEP cavities at CERN. The temperature of the helium bath can be lowered to $1.8 \mathrm{~K}$. In order to stabilise the temperature between $4.2 \mathrm{~K}$ and $1.8 \mathrm{~K}$, a manostat (Oxford Instruments) was integrated in the pumping line to stabilise the pressure in the cryostat. Therefore, we were able to reach a temperature stability of $\approx 0.1 \mathrm{mK}$ at $1.8 \mathrm{~K}$, which corresponds to a pressure variation of about \pm 0.1 mbar.

The critical temperature $T_{\mathrm{c}}$ of the niobium sample has been measured at a constant helium bath temperature $\left(T_{\text {bath }}\right)$ of $1.85 \mathrm{~K}$ by heating the sample with a known power. $T_{\mathrm{c}}-$ defined as the temperature at which the lock of the $\mathrm{rf}$ generator was lost - was equal to $9.15 \pm 0.02 \mathrm{~K}$. In addition, the thermal conductivity $\lambda(\mathrm{T})$ has been investigated between $1.8 \mathrm{~K}$ and $4.2 \mathrm{~K}$ by heating the sample with the dc heater and measuring the temperature gradient along the niobium cylinder. The determined $\lambda(4.2 \mathrm{~K})=6.9 \pm 0.7 \mathrm{~W} / \mathrm{mK}$ which corresponds to $R R R(4.2 \mathrm{~K}) \approx 28$ is in good agreement with the expected value.

The expected BCS surface resistance can be calculated with standard computer codes ${ }^{9}$. Using a London penetration depth $\lambda_{\mathrm{L}}=27 \mathrm{~nm}$, a coherence length $\xi_{0}=36 \mathrm{~nm}$, and a mean free path $\ell=$ $80 \mathrm{~nm}$ together with the measured $T_{\mathrm{c}}$ and $R R R$ one finds $R_{\mathrm{BCS}}(4.2 \mathrm{~K})=60 \pm 4 \mathrm{n} \Omega$.

The surface resistance $R_{\mathrm{s}}(\mathrm{T})$ of the $\mathrm{Nb}$ sample has been measured by using a rf-dc compensation method. Therefore, the temperature of the quadrupole resonator was stabilised at $1.85 \mathrm{~K}$ and the dc heater was switched on to increase the sample temperature by a well defined $\Delta T$ with $\Delta T_{\min } \cong$ $2.9 \mathrm{mK}$. When the thermal equilibrium - defined as no temperature change $(\Delta T<2.9 \mathrm{mK})$ within one minute - was reached, the heater was switched off and the dissipated heater power $P_{\text {diss }}$ was compensated by switching on and increasing the $\mathrm{rf}$ power until the condition $T_{\text {sample }}$ (rf) $=T_{\text {sample }}(\mathrm{dc})$ was fulfilled. By measuring $P_{\text {diss }}, P_{\mathrm{t}}, \omega$, and $\tau$ one gets with equation (10) the sample surface resistance $R_{\mathrm{S} \text {,sample }}(\mathrm{T})$. The results are shown in Fig. 3. The data have been analysed using $R_{\mathrm{s}}(\mathrm{T})=R_{\mathrm{BCS}}(\mathrm{T})+R_{\text {res }}$ and $\alpha=\Delta / k_{\mathrm{B}} T_{\mathrm{c}}$ as well as the residual resistance were fitted with a modified computer code already mentioned above. The obtained value of $\alpha=1.82$ 
\pm 0.01 is in good agreement with literature values ${ }^{10}$, and the residual resistance of $R_{\text {res }}=19.0 \pm$ $0.3 \mathrm{n} \Omega$ is a reasonable value for bulk niobium.

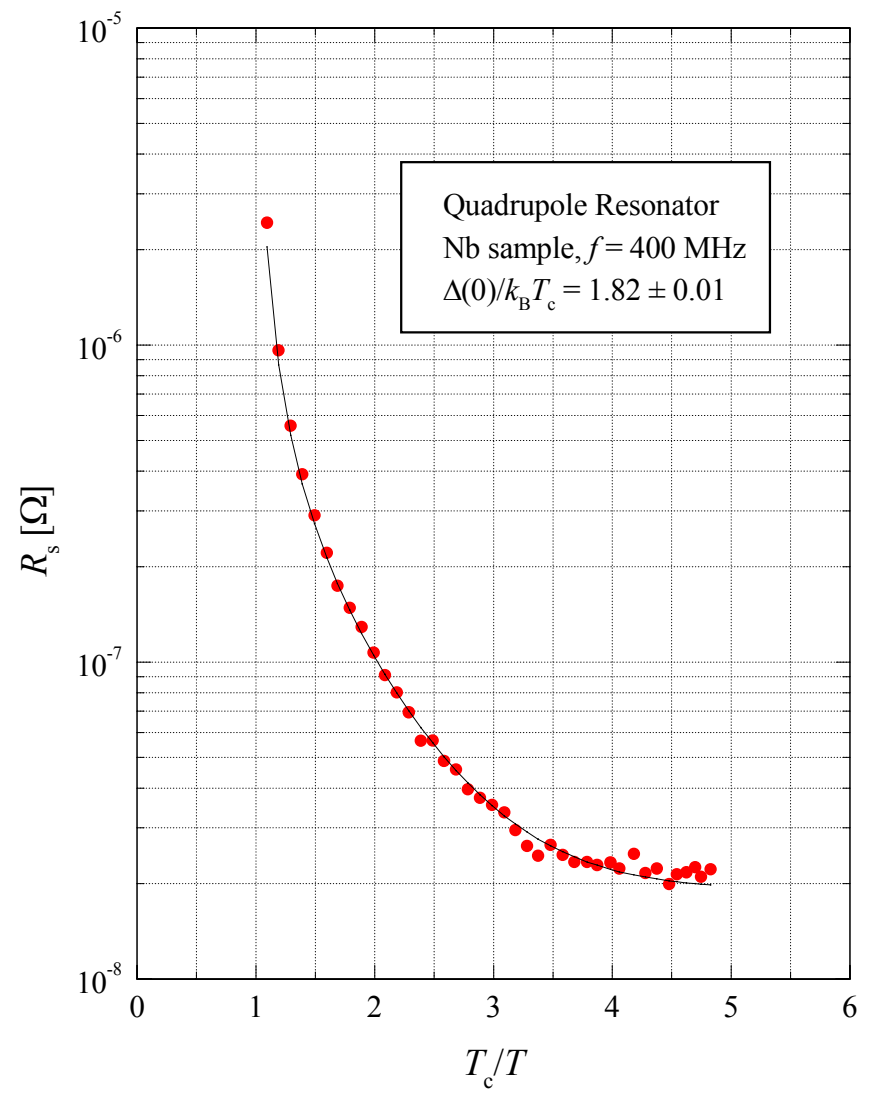

Figure 3: Surface resistance $R_{\mathrm{s}}$ of a reactor-grade bulk niobium sample as a function of $T_{\mathrm{c}} / T$. The experimental points are compared to the fit result (with fixed $\lambda_{\mathrm{L}}, \xi_{0}, \ell$, and $T_{\mathrm{c}}$ ).

The dependence of the surface resistance on the applied field $H_{\mathrm{rf}}$ was measured at different temperatures between $2.3 \mathrm{~K}$ and $4.2 \mathrm{~K}$ by using the compensation method and keeping the temperature of the quadrupole resonator at $2.1 \mathrm{~K}$. The data - shown in Fig. 4 - have been analysed by factorising the surface resistance as

$$
R_{\mathrm{s}}\left(T, H_{\mathrm{rf}}\right)=R_{\mathrm{BCS}}(T) \times\left(1+\alpha_{1} H_{\mathrm{rf}}+\alpha_{2} H_{\mathrm{rf}}^{2}\right)+R_{\mathrm{res}}
$$

With the obtained value of $\alpha_{1}=0$ and $\alpha_{2}=1.32 \times 10^{-5} \pm 1.2 \times 10^{-6}$ one can calculate that $R_{\mathrm{BCS}}$ at $400 \mathrm{MHz}$ increases by $50 \%$ between $0 \mathrm{mT}$ and $\approx 19 \mathrm{mT}$. This result is at variance with the value of $35 \mathrm{mT}$ obtained at $1.5 \mathrm{GHz}^{11}$.

The above results were analysed to determine the obtained resolution for the surface resistance measurements. For the time being, our calorimetric system can measure temperature changes of $\Delta T=2.9 \mathrm{mK}$. This is simply due to the resolution limitation $(100 \mu \mathrm{V}$ in the $2 \mathrm{~V}$ range $)$ of the voltmeter used in the data acquisition unit. Systematic measurements with the dc heater at $4.2 \mathrm{~K}$ have shown that this temperature change of $2.9 \mathrm{mK}$ corresponds to $40 \mu \mathrm{W}$ for the dissipated power which is presently our detection limit at $4.2 \mathrm{~K}$. At a peak field of $H_{\mathrm{rf}}(4.2 \mathrm{~K}) \cong 25 \mathrm{mT}$ and a surface resistance of $R_{\mathrm{s}} \cong 138 \mathrm{n} \Omega$ (see Fig.4), our calorimetric detection limit for $R_{\mathrm{s}}$ is about $0.16 \mathrm{n} \Omega$. Using a more sensitive voltmeter this value can be at least reduced by a factor 10 
which would result in $\Delta R_{\mathrm{S}}<0.02 \mathrm{n} \Omega$. Nevertheless, the already achieved resolution of $0.16 \mathrm{n} \Omega$ at $25 \mathrm{mT}$ and $4.2 \mathrm{~K}$ is by far good enough to investigate all presently known superconductors with the niobium quadrupole resonator.

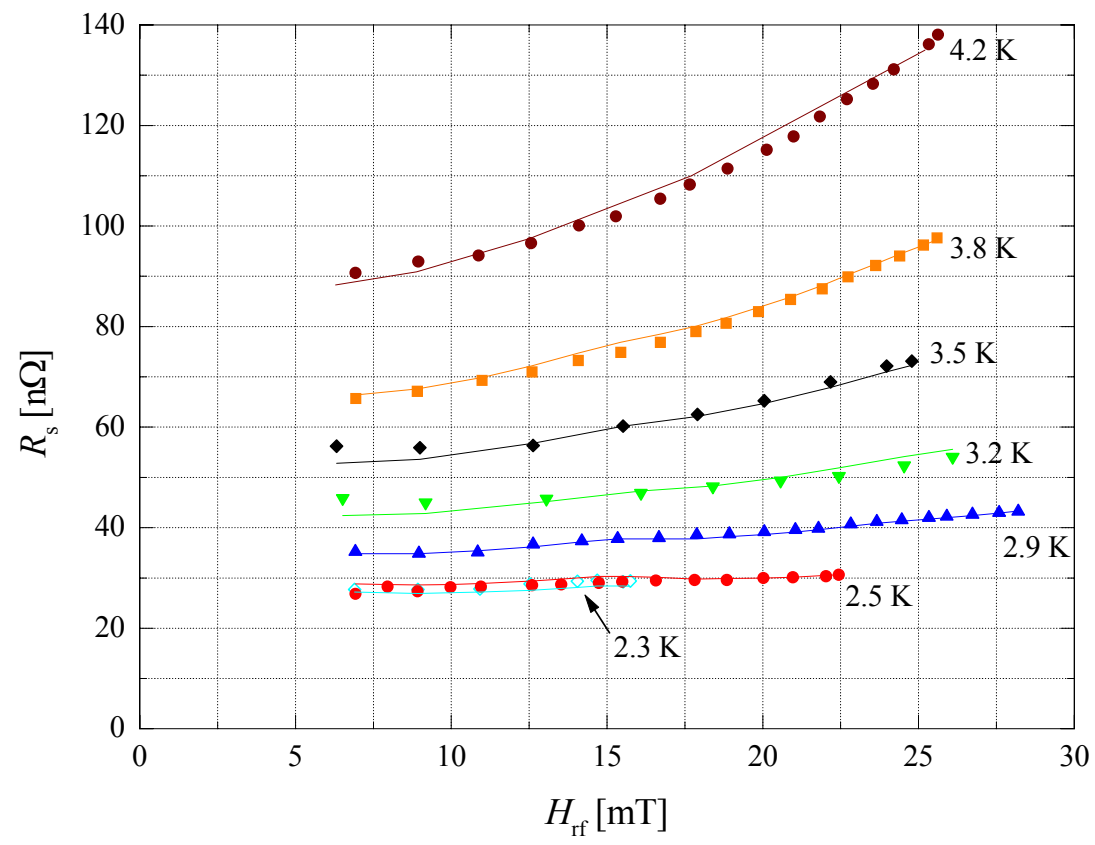

Figure 4: Surface resistance $R_{\mathrm{S}}$ of a reactor-grade bulk niobium sample as a function of applied rf field $H_{\mathrm{rf}}$ measured at $2.3 \mathrm{~K}, 2.5 \mathrm{~K}, 2.9 \mathrm{~K}, 3.2 \mathrm{~K}, 3.5 \mathrm{~K}, 3.8 \mathrm{~K}$, and $4.2 \mathrm{~K}$. The experimental points are compared to the fit results (lines).

\section{Conclusion}

With the niobium quadrupole resonator we have designed a new instrument to study the surface resistance of superconducting samples at $400 \mathrm{MHz}$. First tests with a $75 \mathrm{~mm}$ diameter bulk niobium sample have demonstrated that the rf-dc calorimetric compensation method is sensitive enough to measure changes in the surface resistance which are less than $0.2 \mathrm{n} \Omega$ at $25 \mathrm{mT}$. Therefore, further measurements with $\mathrm{Nb} / \mathrm{Cu}$ or other materials like $\mathrm{Nb}_{3} \mathrm{Sn}$ are very promising.

\section{Acknowledgements}

The realisation of the quadrupole resonator was only possible with the help and assistance of many different people at CERN. Firstly, we would like to thank C. Benvenuti, D. Bloess, D. Boussard, P. Darriulat, J. Tückmantel, and W. Weingarten for many fruitful discussions and suggestions. Secondly, we are grateful for the technical support of H. Preis who helped us in various vacuum and cryogenic aspects of the experimental set-up. Furthermore, we would like to thank S. Bauer and S. Forel for special chemical treatments of the niobium resonator and the sample. Finally, the authors would also like to thank all members of the SL/RF group who contributed to the manufacturing of this new instrument. 


\section{References}

${ }^{1}$ L. Abelmann, C. Lodder, Thin Solid Films 305, 1 (1997).

2 J.M. Laurent, H. Padamsee, CERN/EF/RF 83-2 (1983).

${ }^{3}$ C. Liang, L. Phillips, R. Sundelin, Rev. Sci. Instr. 64, 1939 (1993).

${ }^{4}$ E. Mahner, W. Weingarten, Proc. 8th Workshop on RF Superconductivity,Vol.2, 316, Editor: V. Palmieri, A. Lomdardi, Abano Terme (Padova), Italy 1997.

5 J. Bardeen, L.N. Cooper, J.R. Schrieffer, Phys. Rev. 108, 1175 (1957).

${ }^{6}$ J. Halbritter, Z. Physik 266, 209 (1974).

${ }^{7}$ H. Padamsee, J. Knobloch, T. Hays, $R F$ Superconductivity for Accelerators (1998), John Wiley $\&$ Sons, Inc., ISBN 0-471-15432-6, and references therein.

${ }^{8}$ E. Mahner, G. Müller, H. Piel, N. Pupeter, J. Vac. Sci. Technol. B 13, 607 (1995), E. Mahner, PhD thesis WUB-DIS 95-7, Universität Wuppertal, Germany, July 1995.

9 J. Halbritter, Externer Bericht 3/70-6, Kernforschungszentrum Karlsruhe, Karlsruhe, Germany, June 1970

${ }^{10}$ J. Halbritter, Z. Physik 238, 466 (1970).

${ }^{11}$ C. Benvenuti, S. Calatroni, I.E. Campisi, P. Darriulat, M.A. Peck, R. Russo, A.-M. Valente, Physica C 316, 153 (1999). 